$63^{\text {ème }}$ Congrès de la SFCO, 02004 (2015)

DOI:10.1051/sfco/20156302004

(C) Owned by the authors, published by EDP Sciences, 2015

\title{
COMMUNICATION
}

\section{Dégagement chirurgico-orthodontique des canines mandibulaires Etude rétrospective à propos de 30 cas}

\author{
Bridonneau $\mathrm{T}^{*, * *}$, Niniss $\mathrm{H}^{* * *}$, Quinque $\mathrm{E}^{*, * *}$, Sebe $\mathrm{A}^{* * * *}$, Bahi-Gross $\mathrm{S}^{*, * *, * * * * *}$ \\ * Pôle de médecine et Chirurgie Bucco-Dentaires, UF de Chirurgie Buccale-Implantologie, CHRU de \\ Strasbourg \\ ** Département de Chirurgie Orale, Faculté de Chirurgie Dentaire de Strasbourg, Strasbourg \\ *** Pôle de Médecine et Chirurgie Bucco-Dentaires, UF D’Odontologie Conservatrice, CHRU de Strasbourg \\ **** Pôle de Médecine et Chirurgie Bucco-Dentaires, UF d'Orthopédie Dento-Faciale, CHRU de Strasbourg \\ ***** Cabinet de Chirurgie Orale, Clinique des Diaconeses, Strasbourg
}

L'inclusion de la canine mandibulaire est une pathologie qui affecte $0,2 \%$ de la population générale Borghetti et al. 2008). Elle est dans la majorité des cas unilatérale, le plus souvent à droite (Yavuz et al. 2007) mais peut se présenter associée à une inclusion de la canine maxillaire, voire dans le cadre d'inclusions multiples bilatérales ou multiples dans le cas d'un syndrome.

Afin d'analyser plus précisément ces inclusions, une étude rétrospective sur 26 patients traités pour la prise en charge de 30 inclusions a été conduite sur une durée de 4 ans avec un suivi moyen de 3,5 ans.

Les critères d'inclusions étaient les suivants : Absence d'antécédents médico-chirurgicaux, présence d'une ou plusieurs canines incluses selon la définition de l'AFNOR. 12 paramètres ont également été étudiés (localisation, âge, sexe, nombre d'inclusion, étiologie, position, type de lambeau réalisé, localisation du bouton de traction, type d'imagerie réalisée, actes associées, nécessité de recoller le bouton de traction, échecs éventuels).

Sur les 26 patients, 4 présentaient une inclusion bilatérale (15,3\%) et 22 des inclusions unilatérales (84,6 \%). 57,6 \% étaient de sexe féminin et 42,3\% de sexe masculin. L'analyse de la position de la canine mandibulaire dans le plan sagittal a été effectuée à partir de l'examen clinique et d'éléments radiographiques. 96,6 \% des canines étudiées étaient incluses en position vestibulaire et seule 1 canine était en position linguale. Selon la classification de Mupparapu (2012), $23 \%$ des dents avaient une position oblique dans la région symphysaire (type 1), $23 \%$ étaient mésio-angulées sans franchissement de la ligne médiane, $6 \%$ étaient disto-versées, $10 \%$ étaient en position horizontale, $30 \%$ en position verticale (type 2), et $6 \%$ en position verticale entre les incisives centrales (type 5 ).

Toutes les canines étudiées ont bénéficié d'une prise en charge chirurgicale par le même opérateur et d'un suivi orthodontique par divers praticiens spécialistes en orthopédie dento-faciale. Sur l'ensemble de l'échantillon, 88 \% des canines ont retrouvé une occlusion fonctionnelle, 3 canines ont été avulsées au cours du traitement orthodontique en raison, dans un cas, d'une rétention liée à une courbure radiculaire et dans les 2 autres d'une ankylose.

This is an Open Access article distributed under the terms of the Creative Commons Attribution License 4.0, which permits unrestricted use, distribution, and reproduction in any medium, provided the original work is properly cited. 
Cette présentation est l'occasion de faire le point sur le dégagement des canines mandibulaires en reprenant, à travers les cas d'inclusions traitées, les différentes options chirurgicales, mais également d'étudier le devenir clinique de ces dents confronté aux données de la littérature.

La prise en charge d'une canine mandibulaire incluse illustre bien la collaboration entre le chirurgien oral et l'orthodontiste.

Nom et adresse du conférencier

Thomas BRIDONNEAU

Unité Fonctionnelle de Chirurgie Buccale-Implantologie

Pôle de médecine et chirurgie bucco-dentaires CHRU de Strasbourg

1 Place de l'Hôpital

67000 Strasbourg (France)

thomas.bridonneau@gmail.com 